\title{
Molecular functional imaging in personalized clinical oncology: The road less traveled
}

"I have lived much of my life among molecules. They are good company."

George Wald; Nobel laureate in Physiology and Medicine for his work on retinal physiology.

Molecular imaging is the noninvasive, real-time visualization of biochemical events at the cellular and molecular level within living cells, tissues, and/or intact subjects. ${ }^{[1]}$ This has brought about contrariety in "classical" diagnostic imaging as it sets forth to probe the molecular abnormalities that are the basis of disease rather than to image the end effects of these molecular alterations. ${ }^{[1,2]}$

Recently evolved concept of personalized imaging in oncology has come into sight as a promising approach to address unmet needs of the present cancer medicine. The current driving forces of molecular imaging in the clinical setting worldwide are positron emission tomography (PET) and single photon emission computed radiography (SPECT) imaging. However, these modalities are expensive and provide low spatial resolution for concurrent anatomical correlation; thereby, currently limiting their routine application in developing countries. The modalities used more frequently in day-to-day radiological practice such as magnetic resonance imaging (MRI), computed tomography (CT), and ultrasonography are gaining ground as new avenues for molecular imaging; and in the future are expected to become complete imaging technologies providing a fusion of anatomical as well as functional information. ${ }^{[1,2]}$

However, there is a need to fast-track the translation of recent advances made in molecular imaging research laboratory to the patients' bedside; some campaigns such as "bench to bedside" to achieve this objective are underway. ${ }^{[1,3]}$ The routine teaching to researchers at bench is "ontology recapitulates phylogeny;" however for bedside clinicians, it is "oncology recapitulates polemology, the science of warfare." ${ }^{[4]}$

In vivo molecular imaging has a great potential to impact oncological medicine in the fields of screening, personalized management, and targeted therapy. ${ }^{[1,4]}$ Targeted ultrasound contrast agents, Raman

\begin{tabular}{|l|l|}
\hline \multicolumn{3}{|c|}{ Access this article online } \\
\hline Quick Response Code: & Website: \\
\hline & www.ijmpo.org \\
\cline { 2 - 2 } & \\
\hline
\end{tabular}

spectroscopy, and magnetic resonance spectroscopy; nanotechnology in the form of gold nanoparticles for targeted head and neck cancer CT imaging; MRI cell tracking using 19 fluoride and iron oxide; and fusion techniques such as PET-MRI, etc., are the various molecular imaging applications either currently in practice or under investigation. ${ }^{[1]}$

Imaging features can quantify the spatial variation in architecture and function of individual tumors and can approximately capture measurements of blood flow, hypoxia, metabolism, cell death, and other phenotypic features. ${ }^{[4-6]}$ This robust identification of genotype and phenotype features and establishing their correlation based on relevant clinical questions is called "radiomics" and it challenges the concept "one size fits all." This "radiomics" signature analysis not only helps in prognosticating tumors but also captures intratumor heterogeneity associated with underlying gene-expression patterns. ${ }^{[1,4,7]}$ Recent studies have found that it may have a significant impact in clinical practice and will provide an unprecedented opportunity to improve personalized cancer treatment at low-cost. ${ }^{[1,2]}$ For example, there has been a tremendous advancement in breast cancer management with development of epidermal growth factor receptor (EGFR) and human epidermal growth factor receptor 2 (HER2)/ neu therapies, rapamycin which is mammalian target of rapamycin (mTOR) target and other anti-angiogenic therapies. Maximum in vivo success has been seen in the HER2 therapy that has been validated using the in vivo molecular functional imaging. ${ }^{[5]}$ The use of companion targeted delivery known as "theranostics" is also gaining ground few specific examples being molecular interventional oncoradiology using nanoparticles tagged antibodies and PET radionuclide-based molecular targeted therapies. ${ }^{[1,8]}$

The recently published review article by Mahajan et al. has raised a challenging question of whether the present day radiological modalities can be integrated to the

This is an open access article distributed under the terms of the Creative Commons Attribution-NonCommercial-ShareAlike 3.0 License, which allows others to remix, tweak, and build upon the work non-commercially, as long as the author is credited and the new creations are licensed under the identical terms.

How to cite this article: Mahajan A, Desai S, Kawthalkar AS, Thakur $\mathrm{MH}$. Molecular functional imaging in personalized clinical oncology: The road less traveled. Indian J Med Paediatr Oncol 2016;37:1-3. 

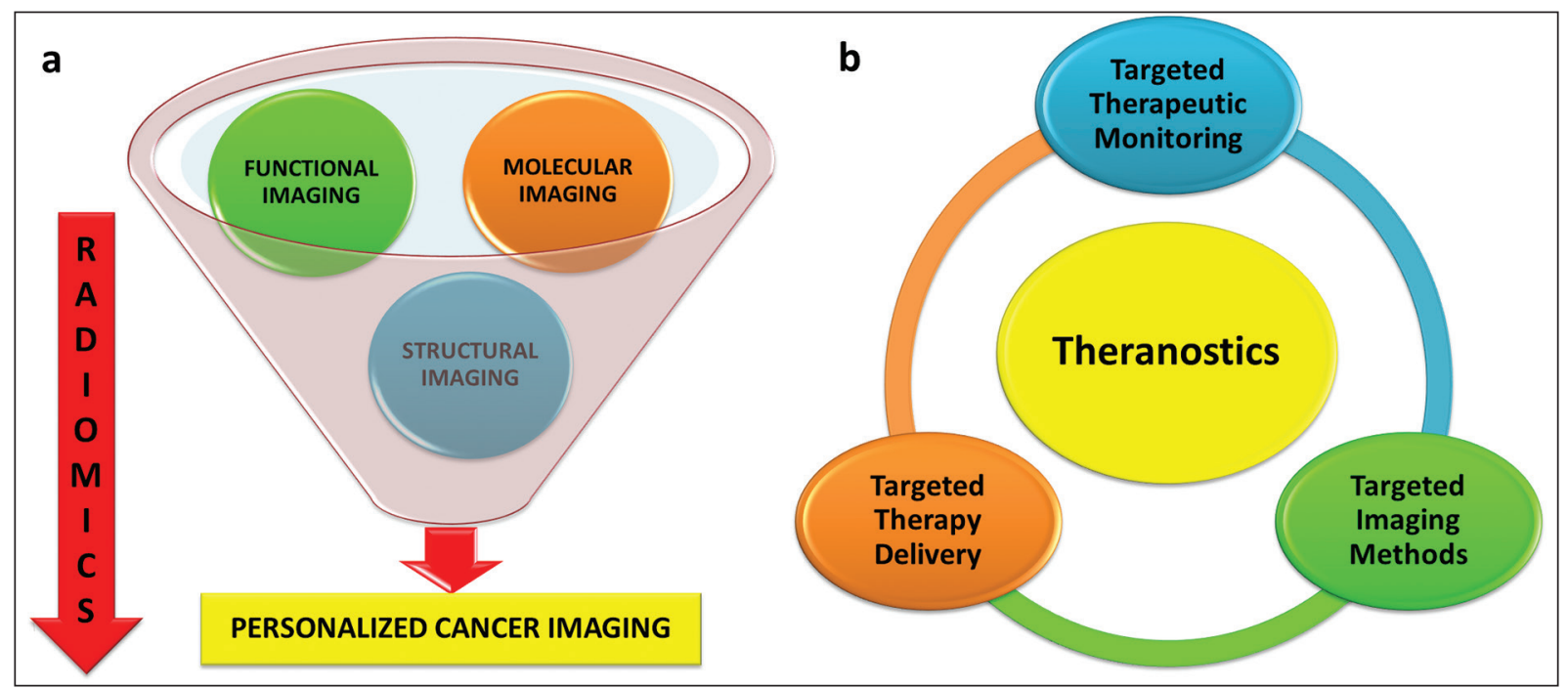

Figure 1: A comprehensive approach to (a) personalized cancer imaging and (b) theranostics

molecular functional imaging for studying the mainstream translational and molecular cancer research and, will it eventually translate the available biological knowledge into clinical practice [Figure 1]. ${ }^{[1]}$ In the present era of limited research budgets and tight reimbursement policies, it is mandatory that the molecular functional imaging is developed by research-driven institutes, industrial collaboration, and government funding. One such example is the 1998 Food and Drug AdministrationNational Institutes of Health (FDA-NIH) criteria which gives the following guideline for assessing the ability of a biomarker at five levels: ${ }^{[9,10]}$

a. To measure biologic activity of disease.

b. To provide diagnosis, differential diagnosis, and prognosis of diseases.

c. To assist therapy development and evaluation.

d. To substitute for clinical endpoints as surrogate endpoints.

e. To guide treatment option and monitor responses.

Many researches in the field of development of molecular functional imaging techniques have failed to show clinical applicability. One such example is the use of F-18 FDOPA PET or I-123 CIT SPECT for neuroimaging, which evaluated as per FDA-NIH biomarker criteria, failed after the third level of testing and did not reveal a clinical endpoint or helped to guide treatment. ${ }^{[1]}$

To conclude, integrating molecular imaging functionalities into therapy is expected to grow significantly in the future and may fulfill the promise of personalized cancer management "to deliver the right care to the right patient at the right time." It is imperative to foresee research advances that should find solutions to provide diagnostic as well as therapeutic molecular imaging services for the general population at affordable prices, and make them widely accessible since they are the next frontier in our battle against personalized cancer management.

\section{Acknowledgments}

I offer my sincere thanks to Dr. Gary Cook and Dr. Vicky Goh, Department of Imaging Science and Biomedical Engineering, Kings College London, UK, for their constant guidance and support.

\section{Financial support and sponsorship} Nil.

\section{Conflicts of interest}

There are no conflicts of interest.

Abhishek Mahajan, Subhash Desai, Ameya Shirish Kawthalkar, Meenakshi Haresh Thakur Department of Radiodiagnosis, Tata Memorial Centre, Mumbai, Maharashtra, India.

Address for correspondence: Dr. Abhishek Mahajan,

Department of Radiodiagnosis, Tata Memorial Hospital, Dr. E Borges Road, Parel, Mumbai, Maharashtra, India. E-mail: drabhishek.mahajan@yahoo.in

\section{REFERENCES}

1. Mahajan A, Goh V, Basu S, Vaish R, Weeks AJ, Thakur MH, et al. Bench to bedside molecular functional imaging in translational cancer medicine: To image or to imagine? Clin Radiol 2015;70:1060-82.

2. Dhingra VK, Mahajan A, Basu S. Emerging clinical applications of PET based molecular imaging in oncology: The promising future potential for evolving personalized cancer care. Indian J Radiol Imaging 2015;25:332-41.

3. Kalia M. Biomarkers for personalized oncology: Recent advances and future challenges. Metabolism 2015;64 3 Suppl 1:S16-21.

4. Aerts HJ, Velazquez ER, Leijenaar RT, Parmar C, Grossmann P, Carvalho S, et al. Decoding tumour phenotype by noninvasive imaging using a quantitative radiomics approach. Nat Commun 2014;5:4006. 
5. O'Connor JP, Jackson A, Asselin MC, Buckley DL, Parker GJ, Jayson GC. Quantitative imaging biomarkers in the clinical development of targeted therapeutics: Current and future perspectives. Lancet Oncol 2008;9:766-76.

6. Mahajan A, Engineer R, Chopra S, Mahanshetty U, Juvekar $\mathrm{SL}$, Shrivastava SK, et al. Role of 3T multiparametric-MRI with BOLD hypoxia imaging for diagnosis and post therapy response evaluation of postoperative recurrent cervical cancers. European Journal of Radiology Open, 3, 2016. p. 22-30.

7. Mahajan A, A V Moiyadi, R Jalali, E Sridhar. Radiogenomics of glioblastoma: a window into its imaging and molecular variability. Cancer Imaging 10/2015; 15 (Suppl 1): p. 14. DOI:10.1186/1470-7330-15-S1-P14.
8. Fleuren ED, Versleijen-Jonkers YM, Heskamp $\mathrm{S}$, van Herpen CM, Oyen WJ, van der Graaf WT, et al. Theranostic applications of antibodies in oncology. Mol Oncol 2014;8:799-812.

9. Katz R. Biomarkers and surrogate markers: An FDA perspective. NeuroRx 2004;1:189-95.

10. NIH-FDA Conference: Biomarkers and surrogate endpoints: Advancing clinical research and applications. Abstracts. Dis Markers 1998;14:187-334.

11. Ravina B, Eidelberg D, Ahlskog JE, Albin RL, Brooks DJ, Carbon $\mathrm{M}$, et al. The role of radiotracer imaging in Parkinson disease. Neurology 2005;64:208-15.

\section{“QUICK RESPONSE CODE” LINK FOR FULL TEXT ARTICLES}

The journal issue has a unique new feature for reaching to the journal's website without typing a single letter. Each article on its first page has a "Quick Response Code". Using any mobile or other hand-held device with camera and GPRS/other internet source, one can reach to the full text of that particular article on the journal's website. Start a QR-code reading software (see list of free applications from http://tinyurl.com/yzlh2tc) and point the camera to the QR-code printed in the journal. It will automatically take you to the HTML full text of that article. One can also use a desktop or laptop with web camera for similar functionality. See http://tinyurl.com/2bw7fn3 or http://tinyurl.com/3ysr3me for the free applications. 